\title{
Pharmacogenomic Application in HER2-positive Breast Cancer
}

\author{
Ana Guedes ${ }^{1}$, Célia A. Gomes ${ }^{1}$, Sara Martins ${ }^{2}$, Rui Cruz ${ }^{1, *}$ \\ ${ }^{1}$ ESTESC-Coimbra Health School, Polytechnic Institute of Coimbra, Coimbra, District of Coimbra and the Centro Region, Portugal. \\ ${ }^{2}$ DCV-Department of Life Sciences, University of Coimbra, Coimbra, District of Coimbra and the Centro Region, Portugal.
}

How to cite this paper: Ana Guedes, Célia A. Gomes, Sara Martins, Rui Cruz. (2021) Pharmacogenomic Application in HER2-positive Breast Cancer. International Journal of Clinical and Experimental Medicine Research, 5(4), 490-497.

DOI: 10.26855/ijcemr.2021.10.011

Received: August 8, 2021

Accepted: September 2, 2021

Published: October 8, 2021

*Corresponding author: Rui Cruz, ESTESC-Coimbra Health School, Polytechnic Institute of Coimbra, Coimbra, District of Coimbra and the Centro Region, Portugal.

Email: ruic@estescoimbra.pt

\begin{abstract}
Background: Pharmacogenomics elucidates the hereditary basis of inter-individual differences in drug responses. Genetic variation influences an individual's response to a pharmacological treatment. Therefore, pharmacogenomic information can be used to make treatment more effective and safer, allowing a personalised treatment, especially in Oncology. Pharmacogenomics and precision medicine strategies are becoming increasingly prevalent in research and clinical practice. Breast cancer is a heterogeneous disease that can be classified in different subtypes and about $20 \%$ to $25 \%$ of all breast cancers are HER2-positive. Several studies have established the HER2 receptor as an effective target for the treatment of breast cancer. Objective: The present review aims to survey the available drugs that target the HER2 protein and to elucidate the way in which pharmacogenomics allows to adapt the therapeutic according to the patient's genetic profile. Methods: A search was conducted using MEDLINE/PubMed and B-on databases. Data were extracted with specific keywords and the inclusion criteria for assessing the studies published between 2013 and 2020. Results: After evaluating the several studies, we included 12 studies in this review. The trastuzumab was the first drug for anti-HER2 therapy and is a reference drug targeting breast cancer. With knowledge ever deeper the genetic patient profile, new drugs have also emerged, like pertuzumab, lapatinib and neratinib, targeting new molecular sites with a different safety and efficacy profile. These drugs can be used alone in combination therapy, which increases the chances of therapeutic success. Conclusion: The personalized medicine based on pharmacogenomic biomarkers allows treatment decisions to be made based on the genotyping of tumors and genetic profiles. Targeting therapy for genetic changes is a promising approach that improves clinical outcomes, in terms of response rates, survival, safety and costs.
\end{abstract}

\section{Keywords}

Pharmacogenomics, Breast Cancer, HER2 Receptor

\section{Introduction}

Over the last decade, there has been a lot of progress pharmacogenetics, which allowed us to unveil the genetic basis of inter-individual differences in drug response [1].

Pharmacogenetics and pharmacogenomics are responsible for the study of the effect of genetic variations on drug metabolism, as well as the influence that the genome may have on the response to the drug [2,3]. The main purpose 
of pharmacogenomics is to optimise therapy, through the understanding of the underlying biological mechanisms and using genomic contributions in response to treatment in order to predict, individualize and improve the results of therapy [3].

The pharmacogenomics biomarkers that can predict the response to drugs promise an improvement in diagnosis and can be noted in germline biomarkers (due to their influence in pharmacokinetics and pharmacodynamics) and biomarkers in the somatic cancer genome (that modulate the response of cancer cells to the drug) [4].

Access to the individual's genetic profile is becoming increasingly common, providing a better and more informed decision while choosing the sort and dosage of treatment. The storage of genetic data can be useful on choosing future therapeutic regimens, in case of tumor recurrence or progression [5].

Pharmacogenomic information is an important tool for patient characterization and gives the possibility of choosing the most appropriate drug and regimen, especially in oncology [4]. Currently, pharmacogenomics-based strategies comprise new approaches, allowing reducing the toxicity and increasing the efficacy of the drug [2].

Cancer is the second leading cause of death worldwide and causes 1 in 6 deaths [4]. Underlying the formation of neoplasms are somatic mutations that activate oncogenes and inactivate tumor suppressor genes. The analysis of genetic differences between tumor and non-tumor cells aims to unveil the genetic basis that gives cancer cells proliferative capacity and the ability to evade apoptosis. By revealing the molecular basis and identifying variants that can be targeted by approved drugs, it is possible to adapt the therapy to the specific tumor, making treatment more effective and faster [4].

To date, the oncogenic variants that were most identified affect signal transduction systems, metabolic enzymes, cell cycle genes, epigenetic machinery or factors involved in translation and transcription. To exemplify, we have therapies aimed at variants in receptor tyrosine kinases, such as EGFR (also known as HER1), ERBB2 (also known as HER2), KIT, among others [4].

Depending on the type of cancer and the nature of said polymorphisms, the use of targeted therapies that interfere with the genetic products found exclusively in cancer cells is recommended. Nevertheless, even in patients who have apparently a predictable positive response to targeted therapy, drug resistance may arise due to the acquisition of additional mutations [4].

Many platforms are already commercially available and are more and more used in clinical trials, allowing the analysis of the genomic DNA of different tumors to verify association between genetic variability and the success of the anti-cancer therapy in question. This approach is guaranteed for the drugs in which the presence of mutations in the genes coding for the receptors will be determinant to the clinical success of the inhibitor in question [4].

In 2018, more than 2 million $(2,088,849)$ new cases of breast cancer in both sexes were registered [6]. Worldwide, breast cancer is the fifth leading cause of death in women and represents a health issue due to the lack of effective therapeutic regimens that can be applied to all groups [2]. It is a heterogeneous disease, which can be divided into several subtypes with different clinical characteristics, distinct sensitivity to therapy and different prognosis [7].

From a molecular point of view, it can be categorized into 5 subtypes: luminal A, luminal B, human epidermal growth factor receptor 2 (HER2), non-luminal and triple-negative (TNBC) [2].

HER2 was discovered over 30 years ago and at the time led to inadequate prognosis [8]. It is common in younger patients and is commonly associated with poorly differentiated high-grade tumors, and is usually overexpressed in $20-25 \%$ of breast cancers [8,9]. The expression or amplification of the HER2 gene in breast cancer is a predictive biomarker. In case of it being HER2-positive, it is predictive for a positive response to a potential treatment with trastuzumab, and prognosis there will be a ill prognosis, if there is a gene overexpression [10]. Several studies show this receptor as an effective therapeutic target for the treatment of breast cancer [2, 7].

HER2 overexpression is assessed by immunohistochemistry (IHC) or by fluorescent in situ hybridization, known as FISH [9].

A better understanding of the pathobiology of HER2 led to the development and approval of the monoclonal antibody trastuzumab, which resulted in a substantial change in the natural history of the disease [8].

Nowadays, there are some treatments that act specifically on this receptor, namely trastuzumab, lapatinib, pertuzumab and trastuzumab emtansine [9]. Neratinib has also received increased interest, as it has shown clinical activity in patients with HER2-positive metastatic breast cancer [2, 11]. In Portugal, only trastuzumab, trastuzumab emtansine and pertuzumab are available [12].

In recent years, there have been made significant advances in the treatment of breast cancer and almost all of them in the area of targeted therapy. There are currently some anti-HER2 therapies that are being developed (for example, margetuximab, trastuzumab deruxtecan-DS-8201a, pyrotinib) and that are able to overcome the resistance mechanisms presented by current drugs in the HER2 signalling pathway [13]. 
The present review aims to survey the available drugs that target the HER2 protein and to elucidate the way in which pharmacogenomics allows to adapt the therapeutic according to the patient's genetic profile.

\section{Methods}

The literature used in this review is available in the PubMed and B-on databases. The keywords selected were "Pharmacogenomics" or "Breast cancer" or "Therapeutics" and "HER2 gene". The inclusion criteria of the studies were adopted: written in English, different type of studies (clinical trials and others) and published between 2013 and 2020.

The selection of articles was carried out in two stages. In the first, we excluded duplicate studies and the title and abstracts were read and those that did not meet the inclusion criteria of the study were excluded. In the second stage, the remaining studies were read in full and, in the same way, those that did not meet the inclusion criteria or the content that were not relevant to this study were excluded. All articles were read in full and assessed for their eligibility, in the end, 12 articles were included in this review.

\section{Results}

\subsection{HER2 receptor}

The HER2 protein has 4 functional domains: extracellular domain (ECD), transmembrane domain (TMD), just membrane domain (JMD) and tyrosine kinase domain (TKD) (Figure 1). The extracellular domain is additionally composed of 4 subdomains [14].

Amplification of the HER2 gene has been identified in more than $14 \%$ of metastasized breast cancers and is associated with increased cell proliferation, angiogenesis, invasion and apoptosis reduction. In HER2 tumors, there are some genes such as BRF2 and DSN1 that undergo amplification or overexpression as oncogenes, conferring a neoplastic advantage [2].

The HER2 oncogenes (HER2, HER2/neu, c-erbB-2) were discovered in 1984 by Weinberg et al. Their aim is to encode the transmembrane receptor tyrosine kinase and is located on chromosome 17. The amplification of the HER2 gene is associated with the multiplication and proliferation of tumor cells, which result from signal transduction mediated by the activation of the phosphatidylinositol 3-kinase pathway (PI3K/AKT) and the mitogen-activated protein pathway (Ras/Raf/MEK/MAPK) [7].

HER2 acts as an important prognosis biomarker in diseases in later stages, and its overexpression has been associated with least good outcomes in breast cancer survival. The amplification and/or overexpression of the HER2 gene were discussed in a review of 107 studies, with the participation of 39,730 patients, who report a total of $22.2 \%$ of the HER2-positive rate and an average relative risk (RR) of 2,74 for global survival (GS), using targeted anti-HER2 therapy [7].

In addition, patients with HER2-positive cancer have a lower rate of response to endocrine therapy and may show to be resistant to non-anthracycline and non-paclitaxel regimens. Therapies targeting HER2 have extremely enhanced survival results for patients with HER2-positive breast cancer [7].

\subsection{Trastuzumab}

Trastuzumab was the first humanized IgG1 monoclonal antibody to be developed against the extracellular domain of the HER2 transmembrane receptor and the first to get approval from the Food Drugs and Administration (FDA) as a drug targeting breast cancer [2, 7, 9]. It can be implemented as monotherapy or in combined therapy [9]. It is usually administered intravenously either weekly or every 3 weeks (cycles of 21 days), depending on the patient's body weight.

The mechanism of action of trastuzumab is linked to the extracellular domain of the HER2 receptor (Table 1), triggering antibody-dependent cell-mediated cytotoxicity (ADCC), blocking of the HER2 receptor's dimerization, inhibition of signal transduction pathways and angiogenesis, interruption of the cell cycle and apoptosis, as well as interfering with DNA repair [7].

Various adverse events have been identified with the use of trastuzumab, such as acute cardiac toxicity in congestive heart failure (CHF), gastrointestinal symptoms, minor hematological deficiencies and pulmonary symptoms. It has an incidence rate of about 1\%, with cardiac toxicity being the limiting factor for the use of this drug [7].

Some studies suggest that the combination of trastuzumab with other chemotherapy regimens attains a better response than chemotherapy alone, with longer survival time, lower mortality rate and longer response time [2, 7]. Other studies, however, reveal that some patients develop resistance to the therapy. 


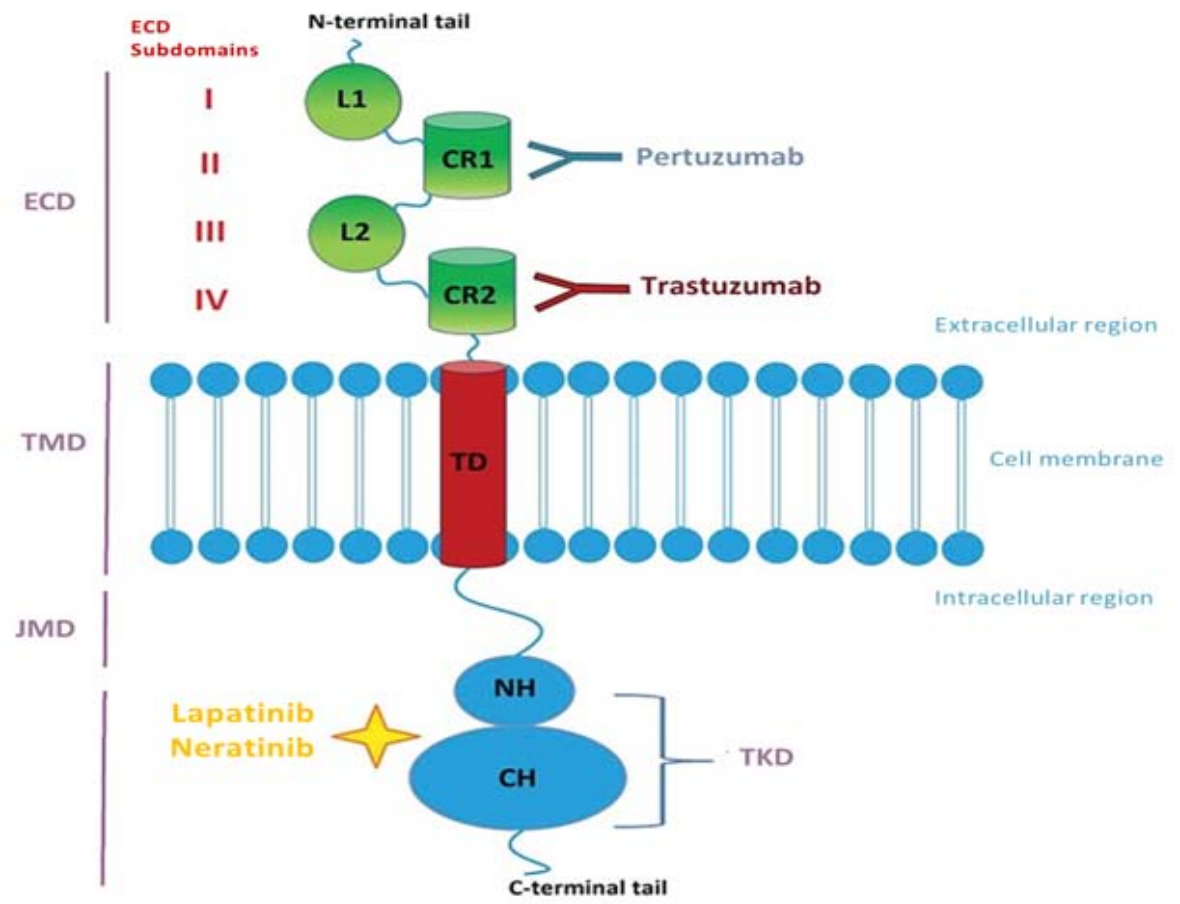

Figure 1. The four functional domains of HER2 protein: extracellular domain (ECD), transmembrane domain (TMD), justmembrane domain (JMD), tyrosine kinase domain (TKD) and the ECD subdomains (Also show the binding site of drugs. Adapted from Perrier et al., 2018 [15]).

Table 1. Therapeutic options for HER2-positive breast cancer [14]

\begin{tabular}{|c|c|c|c|}
\hline Drug & Binding site & Mechanism of action & Therapeutic indication \\
\hline Trastuzumab & $\begin{array}{l}\text { Subdomain IV } \\
\text { of HER2's ECD }\end{array}$ & Blockage of HER2 dimerisation & 1st anti-HER2 therapeutic line \\
\hline $\begin{array}{l}\text { Trastuzumab } \\
\text { emtansina }\end{array}$ & $\begin{array}{l}\text { Subdomain IV } \\
\text { of HER2's ECD }\end{array}$ & $\begin{array}{l}\text { Blockage of HER2 dimerisation and cytotoxicity } \\
\text { induction by toxic agent DM1 }\end{array}$ & Specific cases after treatment with trastuzumab \\
\hline Pertuzumab & $\begin{array}{l}\text { Subdomain II } \\
\text { of HER2's ECD }\end{array}$ & Blockage of HER2 heterodimerisation & $\begin{array}{l}\text { In combination with trastuzumab + docetax- } \\
\text { el/paclitaxel or + chemotherapy }\end{array}$ \\
\hline Lapatinib & TKD & HER2 and EGFR reversible inhibitor & In combination with other anti-HER2 agents \\
\hline Neratinib & TKD & $\begin{array}{l}\text { HER1, HER2 and HER4 receptors irreversible } \\
\text { inhibitor }\end{array}$ & $\begin{array}{l}\text { Adjuvant therapeutic after therapy with trastu- } \\
\text { zumab }\end{array}$ \\
\hline
\end{tabular}

The mechanisms of resistance to trastuzumab appear to be related to the HER2 signalling pathway and associated with activating mutations in PIK3CA, RAS, Src, NFKB and inactivating mutations in PTEN (negative regulatory gene). Other resistance mechanisms identified include overexpression of EGFR and HER-3 and their ligands, interaction with adhesion molecules and incorporation into heterologous receptors (HER-3) [2, 16].

Strategies to overcome resistance to this drug encompass:

- $\quad$ the use of pan-PI3K inhibitor, specific PIK3CA inhibitors, AKT inhibitors and mTOR inhibitors when the resistance results from changes in PIK3CA;

- $\quad$ lapatinib chemotherapy to overcome the high level of p95HER2 (oncogene HER2);

- tyrosine kinase inhibitors or IGF1R monoclonal antibodies to overcome the activation of the IGF1R tyrosine kinase receptor;

- MET inhibitors (proto-oncogene that encodes the hepatocyte growth factor receptor) to overcome changes in the MET gene (mutation and amplification);

- $\quad$ inhibitors of immune checkpoints to overcome the low immune response [2, 16].

Heterogeneity in HER2-positive breast cancers is associated with failure of targeted therapy. Moreover, patients with HER2-positive cancer exhibit molecular changes and mutations in gene expression that can affect prognosis and the response to treatment [2]. 


\subsection{Transtuzumab emtasine}

Trastuzumab emtansine or trastuzumab-DM1 (T-DM1) is an antibody-drug compound that contains trastuzumab covalently linked to DM1 (inhibitor of the maytansinoid microtubules), through the stable thioether ligand MCC (4-[N-maleimidomethyl] cyclohexane-1-carboxylate) [2, 7]. This molecule has 3 characteristics, the anti-HER2 action of trastuzumab, the ability to induce cytotoxicity via the toxic agent DM1 and specific expression in the tissue [7]. This drug has a multifactorial approach, making it a unique chemotherapeutic drug. First, it binds to the extracellular domain of HER2 (Table 1), causing receptor-mediated internalization, followed by lysosomal degradation, which causes extracellular release of DM1, interrupting the formation of microtubules and finally stopping the cell cycle and causing apoptosis [9].

This drug requires high levels of HER2 expression in target cells to be effective [2]. In order to achieve an optimal concentration to induce cell death, trastuzumab and its metabolites need to build up in the cancer cell's cytosol. In this sense, it appears that low intra-tumor levels of HER2 and a weak internalization of the drug are associated with insufficient intracellular expression of DM1, thus representing the main mechanisms of drug resistance $[2,16]$.

Implemented as monotherapy, it is the treatment better aimed at patients with locally advanced or metastatic HER2-positive breast cancer, who have previously undergone treatment with trastuzumab and taxane, either alone or in combination [9].

\subsection{Pertuzumab}

Pertuzumab is a humanized IgG1 monoclonal antibody that binds to the extracellular domain of HER2, subdomain II (Table 1), which is different from the one that which binds trastuzumab [2].

As an HER2 receptor antagonist, it inhibits its extracellular dimerization domain, subsequently inhibiting HER2 ligand-dependent heterodimerization with other members of the HER family, including HER3. In this sense, the main signalling pathways of MAP and P13K [9] are inhibited. Inhibition of these signalling pathways can lead to inhibition of cell proliferation. In addition, pertuzumab mediates ADCC [7].

This drug is indicated as a treatment option for HER2-positive breast cancers in early stages in association with trastuzumab, due to the occurrence of a synergistic effect, and chemotherapy, as a neoadjuvant and adjuvant treatment. It is recommended with first-line treatment for patients with metastatic HER2-positive breast cancer in combination with trastuzumab and docetaxel [7, 9].

\subsection{Lapatinib}

Lapatinib is a tyrosine kinase receptor inhibitor that inhibits HER2 and EGFR auto phosphorylation, it being the only approved intracellular inhibitor for both HER2 and EGFR receptors. It acts as a double reversible inhibitor for these two receptors (Table 1), blocking the MAPK/Erk1/2 and PI3K/AKT pathways. In addition, in in vitro studies, it has been proven to increase cytotoxicity mediated by trastuzumab-dependent cells against breast tumor cells [7].

It is indicated for the treatment of patients with breast cancer with overexpression of HER2, showing synergistic activity when combined with other anti-HER2 agents, such as trastuzumab [7].

A preclinical study revealed that lapatinib inhibits the growth of HER2-positive cancer cells that are resistant to trastuzumab and boosts the apoptotic effect of anti-HER2 agents, suggesting that, in this context, this drug may be effective alone [7].

The combination of lapatinib and trastuzumab represents a strategy to reach both the intracellular and extracellular domains of HER2. However, the question arises as to the resistance acquired due to the presence of overexpression of HER2 in tumor cells, which are especially sensitive to the apoptotic effects of lapatinib. Another resistance mechanism mentioned is the activation of AXL, a membrane receptor that belongs to the tyrosine kinase family [2, $16]$.

\subsection{Neratinib}

Neratinib, an irreversible tyrosine kinase inhibitor, can inhibit the HER1, HER2 and HER4 receptors (Table 1) [2, 7]. It is meant for prolonged adjuvant treatment of patients with early stage breast cancer with positive hormone receptors showing amplification/overexpression of HER2 and who have completed adjuvant therapy based on trastuzumab less than a year before $[12,13]$.

Compared to lapatinib, this drug has shown to have a more valid and consistent inhibitory effect on resistance pathways [7, 13]. 
As assessed in one study, neratinib and trastuzumab have different mechanisms of action, being that their combination may confer a synergistic advantage [7].

\subsection{Implications of HER2 gene mutations in anti-HER2 therapy}

About $20 \%$ to $30 \%$ of inter-individual variability in drug response is attributed to genetic polymorphisms. Epigenetic changes in cancer cells are related to the increase in drug transporters that mediate resistance to chemotherapy [4].

Genetic variations in proteins that play a role in the response to the drug can cause lower or higher exposure to the drug in question, increase the formation of toxic metabolites, increase or decrease interactions with the therapeutic target and even activate the immune system, which can lead to drug-induced idiosyncratic toxicity [4].

With the use of new generation sequencing (NGS), it is possible to predict the effect of variants on the genes and consequently predict the effect on the response to the drug [4]. In addition, the analysis of tumor DNA allows not only to characterize the tumor pharmacogenomics profile but also to choose the treatment with the greatest probability of success $[1,4]$.

Recently, it has been prompted that the presence of mutations in HER2 interferes with the response to anti-HER2 therapy. The mutations in the HER2 gene occur in about $4 \%$ of breast cancer patients, regardless of HER2 amplification $[14,17]$.

Pre-clinical and clinical studies credit these mutations to a role in resistance to anti-HER2 and endocrine therapy, having the ability to activate the protein, increase oncogenicity and induce phosphorylation [14].

In the study carried out by Gaibar et al. (2020), to identify mutations in the HER2 gene, a total of 11 mutations were found in different types of breast cancer, most of them in exon 19 and 20 of the TKD. These mutations may be an alternative mechanism for activating HER2, affecting sensitivity to anti-HER2 therapy. Of the 11 mutations identified (Table 2), it is known that mutations L755S, V777L, D769Y and K753E are responsible for resistance to trastuzumab [14].

Table 2. HER2 gene mutations and its therapeutic implications [14]

\begin{tabular}{|c|c|c|c|}
\hline Mutation & Exon & Protein Domain & Therapeutic implications \\
\hline L755S & 19 & TKD & $\begin{array}{l}\text { - Resistance to lapatinib e trastuzumab } \\
\text { - Sensitivity to afatinib e neratinib }\end{array}$ \\
\hline V777L & 20 & TKD & $\begin{array}{l}\text { - Resistance to trastuzumab } \\
\text { - Sensitivity to lapatinib, afatinib e neratinib }\end{array}$ \\
\hline D769Y & 19 & TKD & $\begin{array}{l}\text { - Resistance to trastuzumab e lapatinib } \\
\text { - Sensitivity to neratinib }\end{array}$ \\
\hline $\mathrm{D} 769 \mathrm{H}$ & 19 & TKD & - Sensitivity to neratinib, trastuzumab, pertuzumab e lapatinib \\
\hline K753E & 18 & $\mathrm{KD}$ & $\begin{array}{l}\text { - Resistance to lapatinib e trastuzumab } \\
\text { - Sensitivity to neratinib }\end{array}$ \\
\hline I767M & 19 & TKD & - Sensitivity to neratinib, trastuzumab, afatinib e lapatinib \\
\hline R678Q & 17 & JMD & - Sensitivity to neratinib, trastuzumab, afatinib e lapatinib \\
\hline V697L & - & JMD & -- \\
\hline I655V & 16 & TMD & - Contradictory results \\
\hline S310Y/F & 8 & ECD & $\begin{array}{l}\text { - Sensitivity to neratinib e trastuzumab } \\
\text { - Ineffectiveness of Trastuzumab + pertuzumab }\end{array}$ \\
\hline
\end{tabular}

The L755S mutation is the most common mutation in the HER2 gene and is considered a hotspot mutation. In pre-clinical studies, resistance to lapatinib has been demonstrated in patients with HER2-positive breast cancer, in which the gene is overexpressed $[14,17,18]$. This mutation also induces resistance to trastuzumab alone and in combination with lapatinib or pertuzumab, as demonstrated by in vitro models. This resistance can be overcome using neratinib, which has been shown to be effective in patients with this mutation. Futhermore, in vitro studies have shown sensitivity to the combination therapy of afatinib and neratinib [14].

The V777L mutation is also considered a hotspot mutation and is associated with resistance to trastuzumab and reduced response to lapatinib, afatinib and neratinib [14, 17].

The D769Y and D769H mutations are the most frequent in the HER2 gene, located in exon 19 of the TKD [14, 18]. In vivo studies have shown that neratinib is effective in the presence of these mutations [14, 17]. Additionally, 
some cases have shown resistance to trastuzumab and lapatinib in the presence of the D769Y mutation [14].

The I767M mutation is another hotspot in the HER2 gene and has been identified in patients with HER2-positive breast cancer. In vitro studies have proven sensitivity to therapy with lapatinib, neratinib, afatinib and trastuzumab [14].

In the JMD, 2 mutations were identified: R678Q and V697L. The R678Q mutation demonstrated sensitivity to treatment with trastuzumab, lapatinib, afatinib and neratinib. With respect to the V697L mutation, there is no information available on changes in response to therapy. In TMD, only one mutation was identified: I655V. Here, the influence of this mutation on the response to trastuzumab + chemotherapy therapy in patients with HER2-positive breast cancer is contradictory in several studies. Lastly, in the ECD domain, 2 mutations were identified: S310Y and S310F. In the presence of these mutations, the HER2 protein appears more sensitive to therapy with trastuzumab and neratinib, however the combination of trastuzumab and pertuzumab is ineffective, as demonstrated in an in vitro study [14].

\section{Discussion and Conclusion}

Breast cancer is a complex and heterogeneous disease that affects many women worldwide [2, 7]. HER2-positive breast cancer represents about $20 \%-25 \%$ of all types of breast cancers, as it is very aggressive and there is a high probability of tumor recurrence or progression (metastases), thus it being a concern in research and clinical practice [7-9].

The discovery of the HER2 gene, an understanding of the pathobiology of this receptor and the development of therapies targeting the HER2 receptor resulted in a decline in the morbidity and mortality of breast cancer [8]. Anti-HER2 therapies show very good survival results for patients with HER2-positive breast cancer [7].

Trastuzumab is a reference drug for anti-HER2 therapy that alters the prognosis in patients with HER2-positive breast cancer $[7,9]$. Treatment over the course of one year with trastuzumab is the standard period for adjuvant therapy [7].

The combination of trastuzumab with pertuzumab is recommended through several guidelines and it appears to benefit patients with a high risk of tumor recurrence [7, 9]. Still, 25\% of patients with early stage HER2-positive breast cancer present a recurrence after anti-HER2 therapy. Currently, the first line of treatment for metastatic HER2-positive breast cancer is the combined therapy of trastuzumab + pertuzumab, followed by T-DM1 [7].

Lapatinib and neratinib are also indicated for the treatment of patients with HER2 overexpression/amplification. Nonetheless, it was observed that some of the patients did not respond or develop resistance after some time of treatment [7].

Strategies based on pharmacogenomics endow to overcome difficulties in therapy, such as lack of effectiveness and resistance to some drugs [5, 17].

Genetic analysis of the patient's DNA makes it possible to increase the safety of the treatment in several aspects. Considering the lack of efficacy, the administration of selected therapies based on tumor DNA, allow an increase in the probability of response to a given therapy [5].

Resistance to anti-HER2 therapy remains a major problem, despite the development of new drugs. What is more, the plasticity of tumor cells and the heterogeneity of tumors can contribute to the occurrence of acquired resistance $[4,17]$.

One way to prevent/delay treatment resistance is to detect new mutations and start therapy before the tumor progresses. This is attained by accessing the molecular profile of the tumor tissue or using techniques with circulating tumor DNA $[1,4,17]$.

By accessing the patient's genetic profile, it is possible to choose the most appropriate treatment and regimen, using a genomic biomarker (HER2 receptor) as a guide [4, 5].

Overexpression/amplification of HER2 is associated with a low survival rate. Withal, somatic mutations in the HER2 gene can influence the results of therapy. Still, few mutations have been identified in HER2-positive breast cancer. Most of the identified mutations are classified as oncogenic and/or hotspots. Notwithstanding, for two of the eleven mutations (I767M and K753E), there is still not enough information to classify them [14].

Supplementary studies are needed that relate the presence of somatic mutations in the HER2 gene and its effects on anti-HER2 therapy, in order to improve and individualize therapy, ensuring greater safety and efficacy of drugs.

In conclusion, personalized medicine based on pharmacogenomic biomarkers allows treatment decisions to be made based on the genotyping of tumors and genetic profiles. Targeting therapy for genetic changes is a promising approach that improves clinical outcomes, in terms of response rates, survival, safety and costs. In the future, the practical application of pharmacogenomics shall increase, as major advances have been and are being made to im- 
prove the diagnosis and treatment of breast cancer. Besides providing a better prognosis, faster treatment and fewer adverse effects, it also avoids spending on ineffective treatments and allows for faster recovery of the patient. Although the benefit of using genetic information is evident, the impact of these advances is still limited, and it is necessary to continue studies in this area.

\section{References}

[1] Rodríguez-Vicente, A. E., Lumbreras, E., Hernández, J. M., Martín, M., Calles, A., Otín, C. L., et al. (2016). Pharmacogenetics and pharmacogenomics as tools in cancer therapy. Drug Metab Pers Ther., 2016, 31(1): 25-34.

[2] Jeibouei, S., Akbari, M. E., Kalbasi, A., Aref, A. R., Ajoudanian, M., Rezvani, A., et al. (2019). Personalized medicine in breast cancer: Pharmacogenomics approaches. Pharmgenomics Pers Med., 2019, 12: 59-73.

[3] Filipski, K. K., Mechanic, L. E., Long, R., Freedman, A. N. (2014). Pharmacogenomics in oncology care. Front Genet, 2014, 5(APR): 1-5.

[4] Lauschke, V. M., Milani, L., Ingelman-Sundberg, M. (2018). Pharmacogenomic Biomarkers for Improved Drug Therapy-Recent Progress and Future Developments. AAPS J., 2018, 20(1).

[5] Innocenti, F. (2017). Bringing a genomic perspective to the safety of drug treatment in oncology. F1000Research, 2017, 6: 1-5.

[6] Globocan Observatory, W. (2019). Cancer Today-World. Int Agency Res Cancer [Internet], 2019, 876: 2018-9. Available from: https://gco.iarc.fr/today/data/factsheets/populations/900-world-factsheets.pdf.

[7] Wang, J., Xu, B. (2019). Targeted therapeutic options and future perspectives for HER2-positive breast cancer. Signal Transduct Target Ther [Internet], 2019, 4(1). Available from: http://dx.doi.org/10.1038/s41392-019-0069-2.

[8] Mahtani, R., Hineman, L. (2019). Advances in HER2-Positive Breast Cancer: Novel Therapies and Adverse Event Management. J Adv Pract Oncol [Internet], 2019, 10(2): 136-53. Available from: http://www.ncbi.nlm.nih.gov/ pubmed/31538025\%0A http://www.pubmedcentral.nih.gov/articlerender.fcgi?artid=PMC6750923

[9] Zhang, Y., Somtakoune, S. D., Cheung, C., Listiawan, M., Feng, X. (2016). Therapeutic Application of Pharmacogenomics in Oncology. AAPS J [Internet], 2016, 18(4): 819-29. Available from: http://dx.doi.org/10.1208/s12248-016-9926-x.

[10] Kalia, M. (2015). Biomarkers for personalized oncology: Recent advances and future challenges. Metabolism [Internet]. 2015, 64(3): S16-21. Available from: http://dx.doi.org/10.1016/j.metabol.2014.10.027.

[11] Chan, A., Delaloge, S., Holmes, F. A., Moy, B., Iwata, H., Harvey, V. J., et al. (2016). Neratinib after trastuzumab-based adjuvant therapy in patients with HER2-positive breast cancer (ExteNET): A multicentre, randomised, double-blind, placebo-controlled, phase 3 trial. Lancet Oncol [Internet], 2016, 17(3): 367-77. Available from: http://dx.doi.org/10.1016/ S1470-2045(15)00551-3.

[12] Infarmed. Infomed-Base de dados de medicamentos [Internet]. 2019 [cited 2019 Dec 2]. Available from: http://app7.infarmed.pt/infomed/inicio.php.

[13] Welslau, M., Hartkopf, A. D., Müller, V., Wöckel, A., Lux, M. P., Janni, W., et al. (2019). Update Breast Cancer 2019 Part 5-Diagnostic and Therapeutic Challenges of New, Personalised Therapies in Patients with Advanced Breast Cancer. Geburtshilfe Frauenheilkd, 2019, 79(10): 1090-9.

[14] Gaibar, M., Beltrán, L., Romero-Lorca, A., Fernández-Santander, A., Novillo, A., Selli, C. (2020). Somatic Mutations in HER2 and Implications for Current Treatment Paradigms in HER2-Positive Breast Cancer. J Oncol., 2020.

[15] Perrier, A., Gligorov, J., Lefèvre, G., Boissan, M. (2018). The extracellular domain of Her2 in serum as a biomarker of breast cancer. Lab Investig [Internet]., 2018, 98(6): 696-707. Available from: http://dx.doi.org/10.1038/s41374-018-0033-8.

[16] Gagliato, D. D. M., Leonardo, D., Jardim, F., Pereira, M. S., Hortobagyi, G. N. (2016). Mechanisms of resistance and sensitivity to anti-HER2 therapies in HER2 + breast cancer Introduction : Pathways to Trastuzumab. Oncotarget., 2016, 7(39).

[17] Mishra, R., Hanker, A. B., Garrett, J. T. (2017). Genomic alterations of ERBB receptors in cancer: Clinical implications. Oncotarget, 2017, 8(69): 114371-92.

[18] Wen, W., Chen, W. S., Xiao, N., Bender, R., Ghazalpour, A., Tan Z, et al. (2015). Mutations in the kinase domain of the HER2/ERBB2 gene identified in a wide variety of human cancers. J Mol Diagnostics [Internet], 2015, 17(5): 487-95. Available from: http://dx.doi.org/10.1016/j.jmoldx.2015.04.003. 